\title{
DR5 suppression induces sphingosine-1- phosphate-dependent TRAF2 polyubiquitination, leading to activation of JNK/AP-1 and promotion of cancer cell invasion
}

\author{
You-Take Oh, Ping Yue and Shi-Yong Sun
}

\begin{abstract}
Background: Death receptor (DR5), a well-characterized death domain-containing cell surface pro-apoptotic protein, has been suggested to suppress cancer cell invasion and metastasis. However, the underlying mechanisms have not been fully elucidated. Our recent work demonstrates that DR5 suppression promotes cancer cell invasion and metastasis through caspase-8/TRAF2-mediated activation of ERK and JNK signaling and MMP1 elevation. The current study aimed at addressing the mechanism through which TRAF2 is activated in a caspase-8 dependent manner.

Results: DR5 knockdown increased TRAF2 polyubiquitination, a critical event for TRAF2-mediated JNK/AP-1 activation. Suppression of sphingosine-1-phosphate (S1P) generation or depletion of casapse-8 inhibited not only enhancement of cell invasion, but also elevation and polyubiquitination of TRAF2, activation of JNK/AP-1 activation and increased expression of MMP1 induced by DR5 knockdown.

Conclusions: Both S1P and caspase-8 are critical for TRAF2 stabilization, polyubiquitination, subsequent activation of JNK/AP1 signaling and MMP1 expression and final promotion of cell invasion.
\end{abstract}

Keywords: Death receptor 5, Invasion, S1P, Caspase-8, TRAF2, Polyubiquitination

\section{Background}

Death receptor (DR5; also called as TRAIL-R2 or Killer/ DR5) is a death domain-containing transmembrane cell surface protein. DR5 is well known to mediate apoptosis upon ligation with its ligand or induction of its clustering or aggregation (e.g., with an agonistic antibody or overexpression). This process involves activated DR5 interaction with the adaptor protein, Fas-associated death domain (FADD), which further recruits and activates caspase-8 $[1,2]$.

Despite its well characterized apoptotic function, the precise physiological or pathological role of DR5 in the regulation of human cancer development remains unclear $[3,4]$. Mice deficient in mouse TRAIL death

\footnotetext{
* Correspondence: ssun@emory.edu

Department of Hematology and Medical Oncology, Emory University School of Medicine and Winship Cancer Institute, 1365-C Clifton Road, Clinical Building C3088, Atlanta, GA 30322, USA
}

receptor (mDR; the sole mouse ortholog of human DR4 and DR5) show increased susceptibility to tumorigenesis, such as Myc-driven lymphoma and diethylnitrosamineinduced hepatocarcinogenesis [5]. Moreover, mDR deficiency in mice enhances lymph node metastasis of skin carcinoma [6] and metastasis of lymphoma cells to the liver and lung during c-myc-driven lymphomagenesis [5], suggesting that $\mathrm{mDR}$ may be critical for the negative regulation of tumor metastasis. Studies with human cancer samples have shown a reduced DR5 expression in metastatic lesions of melanoma [7] and in primary head and neck tumors with metastasis and their matching lymph node metastasis [8]. Furthermore, inactivating mutations primarily in the death domain of DR5 were detected in $20 \%$ of tissues from breast cancer patients with lymph node metastasis, but were not found in tissues from breast cancer patients without metastasis [9]. 
The DR5 agonistic antibody lexatumumab robustly suppresses lymph node or lung metastasis in an orthotopic model of triple-negative breast cancer [10]. Our recent study with various human cancer cells clearly shows that genetic knockdown or knockout of DR5 significantly increased cancer cell invasion and metastasis in vivo [11]. These findings support the notion that DR5 may be associated with suppression of cancer metastasis.

However, opposing findings have also been reported. One study suggested that oncogenic K-Ras and its effector, Raf1, can convert death receptors (e.g., Fas and DR5) into invasion-inducing receptors by suppressing the ROCK/LIM kinase pathway, and this is essential for K-Ras/Raf1-driven metastasis formation [12]. Another recent publication reports that $\mathrm{mDR}$ and human DR5 promote K-Ras-driven cancer progression, invasion and metastasis since deficiency of $\mathrm{mDR}$ suppressed tumor growth and metastasis in K-Ras-driven carcinogenesis [13]; however, the results of this group are contradictory to their previous findings using a H-Ras-driven skin carcinogenesis model [6]. Hence, the role of DR5 in the regulation of cancer growth and metastasis remains unclear and needs further investigation.

Our recently study has suggested that, under conditions of DR5 suppression, available FADD and caspase-8 may recruit and stabilize tumor necrosis factor receptorassociated factor 2 (TRAF2), resulting in the activation of ERK and JNK signaling and subsequent AP-1dependent expression and activation of MMPs (e.g., MMP1) and final promotion of invasion and metastasis of cancer cells [11]. However, the association between caspase- 8 and TRAF2 activation is undefined and hence was the focus of the current study.

\section{Methods}

\section{Reagents}

SK1-II was purchased from Echelon Bioscience, Inc (Salt Lake City, UT). The human monoclonal DR5 agonistic antibody, AMG655 (Conatumumab), was supplied by Amgen (Thousand Oaks, CA). Antibodies against K63 and K48 were purchased from Cell Signaling Technology (Danvers, MA). Other antibodies and reagents were the same as described previously [11].

\section{Cell lines and cell culture}

A549, 801C and HEK293T cells were described previously [11]. These cell lines were cultured in RPMI 1640 medium containing $5 \%$ fetal bovine serum at $37{ }^{\circ} \mathrm{C}$ in a humidified atmosphere of $5 \% \mathrm{CO}_{2}$ and $95 \%$ air.

\section{Western blot analysis}

Whole-cell protein lysates were prepared and analyzed by Western blotting as described previously [14]. Protein levels were quantified with NIH Image J software based on band density and were normalized to an internal loading control protein.

\section{Expression constructs and transfection}

HA-Ubiquitin-wild-type (WT), -K63 and -K48 expression constructs and his-Ubiquitin expression plasmid were purchased from Addgene (Cambridge, MA). WT and mutant (C360A) Caspase-8 expression constructs [15] were provided by Dr. K. Vuori (Burnham Institute for Medical Research, La Jolla, CA). Flag-TRAF2 expression construct [16] was provided by Dr. H. Habelhah (University of Iowa, Iowa City, IA). Generally, cells were transfected with the given plasmids using Lipofectamine $^{\mathrm{Tm}} 2000$ (Invitrogen) as instructed by the manufacturer's protocol.

\section{Gene silencing using small interfering siRNA (siRNA) or short hairpin RNA (shRNA)}

Gene silencing was achieved by either transfecting siRNA using HiPerFect transfection reagent (Qiagen, Valencia, CA) following the manufacturer's instructions or infecting cells with lentiviruses harboring a given shRNA. Control (i.e., non-silencing) and DR5-specific siRNAs were described previously [14]. DR5 shRNA in pLKO.1 (TRCN0000005929) was purchased from Open Biosystems (Huntsville, AL). Caspase-8 (sc-29930) and sphingosine kinase 1 (SphK1; sc-44114) siRNAs were purchased from Santa Cruz Biotechnology, Inc. Gene silencing effects were evaluated by Western blot analysis as described above.

\section{Reporter plasmids, transient transfection, and luciferase activity assay}

AP-1 (pAP1-luc) and MMP1 promoter luciferase reporter constructs were described previously [11]. Transient co-transfection of DR5 siRNA, Flag-TRAF2 and $\mathrm{pCH} 110$, a plasmid expressing $\beta$-galactosidase ( $\beta$-gal) with lipefectamine was conducted in 24-well plates followed by luciferase assays as described previously [17]. Luciferase activity was normalized to $\beta$-gal activity, which was measured as described previously [18].

\section{Immunoprecipitation (IP)}

The cells were lysed in RIPA buffer with protease and phosphatase inhibitors. The cell lysates were then incubated with anti-Flag M2, anti-HA agarose or antiTRAF2 (sc-7187; Santa Cruz Biotechnology, Inc) at $4{ }^{\circ} \mathrm{C}$ overnight according to the manufacturer's instruction (for tagged proteins). The beads were then washed four times (5 min each) with the same buffer used for cell lysis and boiled in $2 \times$ SDS sample buffer for $5 \mathrm{~min}$. Samples were then analyzed by SDS-PAGE followed by Western blotting. 


\section{Cell invasion and growth assays}

Measurements for cell invasion and cell numbers were the same as described previously [11].

\section{Statistical analyses}

The statistical significance of differences between two groups was analyzed with two-sided unpaired Student's $t$ tests when the variances were equal or with Welch's corrected $t$ test when the variances were not equal by use of Graphpad InStat 3 software (GraphPad Software, San Diego, CA).

\section{Results}

DR5 knockdown increases TRAF2 polyubiquitination that is important for activation of JNK/AP-1 signaling

In our previous report, we have shown that DR5 knockdown elevated TRAF2 levels and increased AP1 , but not NF- $\mathrm{kB}$, activity [11]. It has been suggested that TRAF2 polyubiquitination, including K63 and K48 polyubiquitination, is required for TRAF2 to activate JNK, but not NF-KB [19]. Therefore, we first determined whether DR5 regulates TRAF2 polyubiquitination. As presented in Fig. 1a, co-transfection of Flag-TRAF2 and Ub-HA led to increased levels of

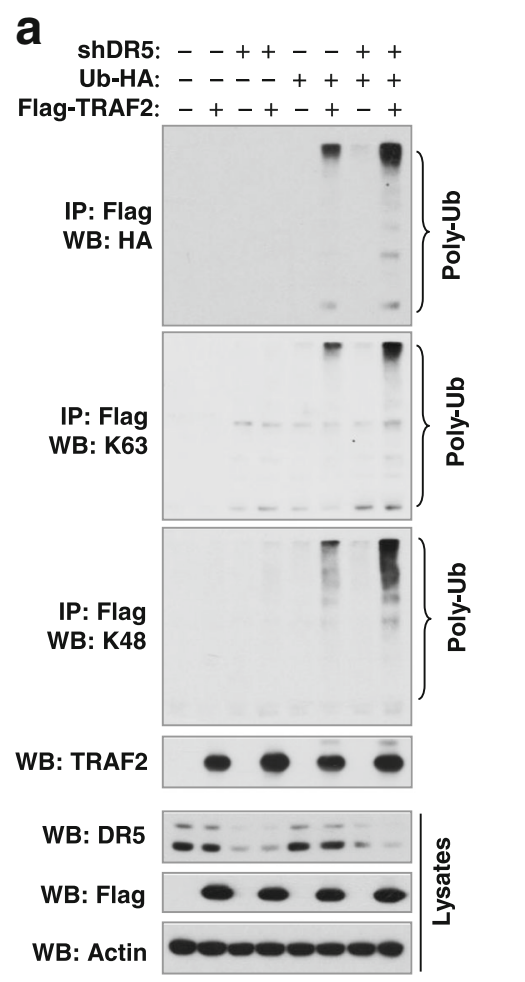

b
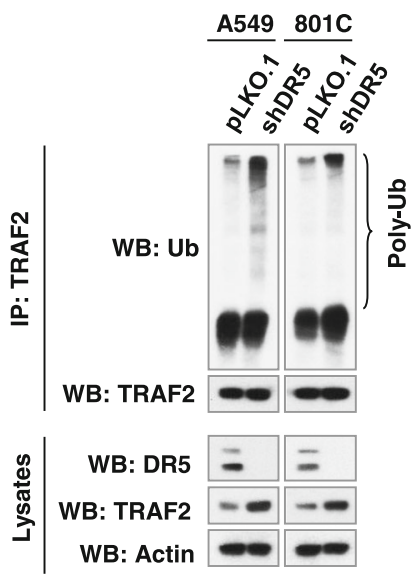

C

C $\square-\mathrm{Ub}-\mathrm{HA} \square+\mathrm{Ub}-\mathrm{HA}$
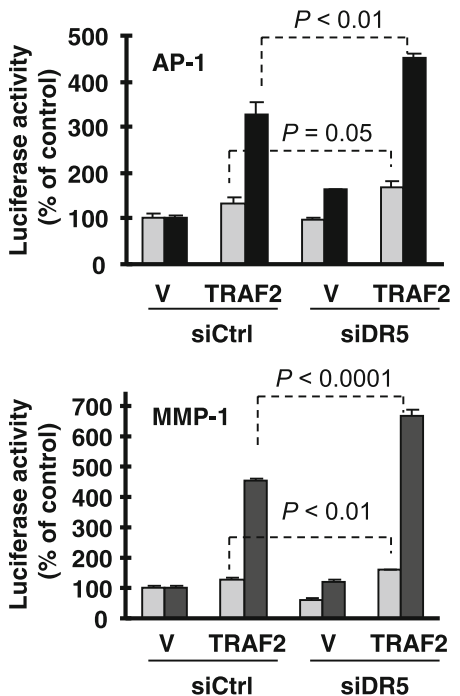

Fig. 1 DR5 knockdown increases TRAF2 polyubiquitination (a and b), which enhances AP-1 transactivation (c), a, HEK293T cells were cotransfected with the indicated plasmids carrying the indicated genes. After $42 \mathrm{~h}$, the cells were lysed for IP with anti-Flag antibody and subsequent Western blotting (WB) for the indicated proteins. The experiments were done twice with identical results. $\mathbf{b}$, Whole-cell protein lysates were prepared from the indicated different cell lines and then subjected to IP with TRAF2 antibody and subsequent Western blotting (WB) for different proteins as indicated. c, HEK293T cells were co-transfected with vector (V) or Flag-TRAF2 plasmid and an control (siCtrl) or DR5 siRNA (siDR5) together with an MMP1 or AP-1 luciferase reporter construct and pCH110 plasmid. After 36 h, the cells were lysed to assay luciferase activity, which was normalized to $\beta$-gal activity. Each column represents a mean \pm SD of triplicate determinations from a representative experiment. The experiments were done twice with similar results 
polyubiquitinated TRAF2 including K63- and K48specific ubiquitination, which were further increased by DR5 knockdown. In DR5-knocked down A549 and $801 \mathrm{C}$ cell lines, increased endogenous TRAF2 polyubiquitination was also detected in comparison with pLKO.1 controls cells (Fig. 1b). These data together suggest that DR5 knockdown increases TRAF2 polyubiquitination. Moreover, we tested whether
TRAF2 polyubiquitination affects AP-1 activity. Cotransfection of Flag-TRAF2 and Ub-HA was much more effective than TRAF2 alone in increasing MMP1 (carrying AP-1 binding site) and AP-1 promoter activity. When DR5 was knocked down, these effects were further significantly enhanced (Fig. 1c). These results suggest that TRAF2 polyubiquitination indeed enhances AP-1 transactivation.

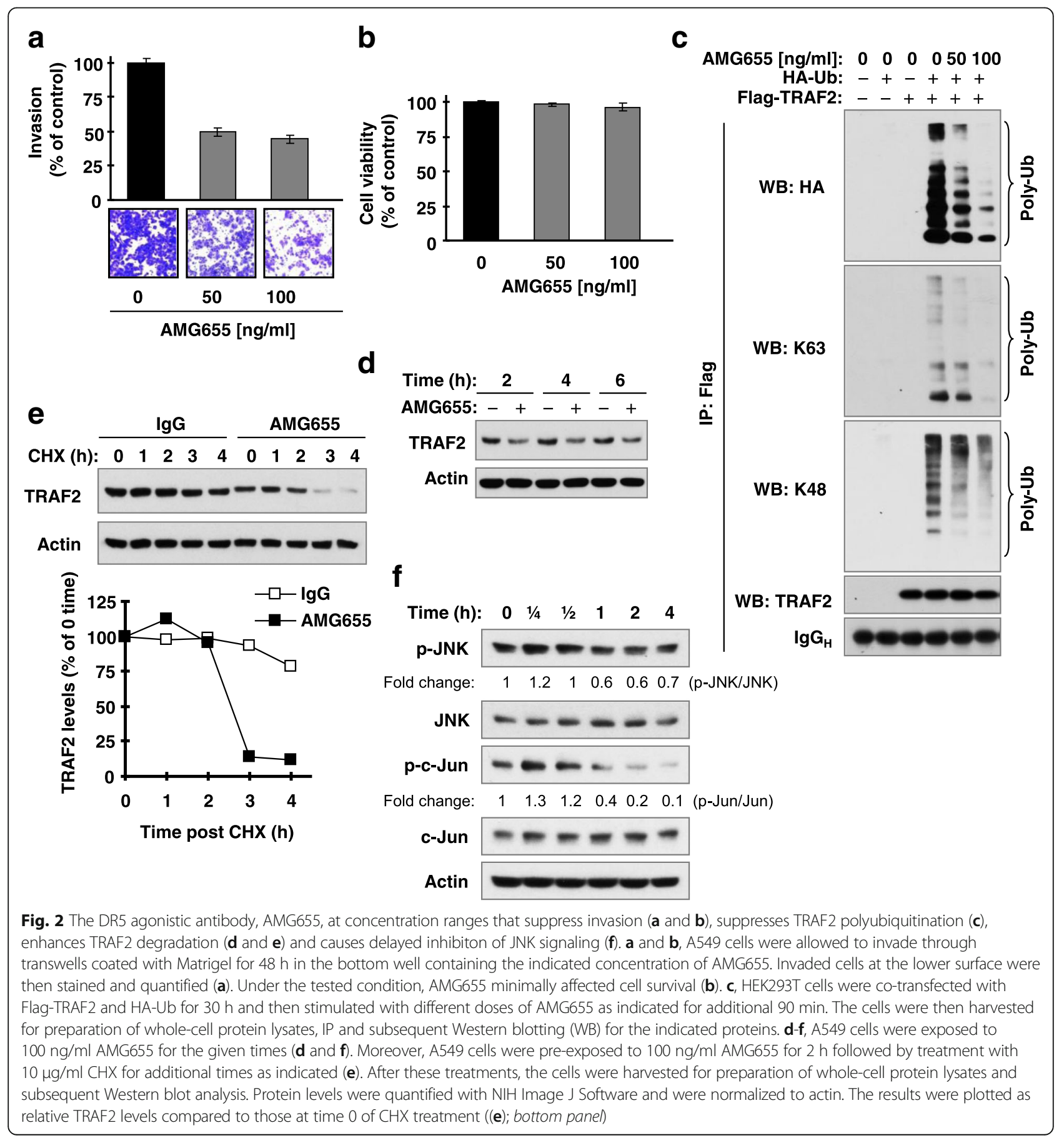


DR5 activation by an agonistic antibody promotes TRAF2 degradation, decreases TRAF2 polyubiquitination, suppresses JNK signaling and inhibits invasion Next we checked the impact of DR5 activation with an agonistic antibody on TRAF2 polyubiquitinaiton. With the concentration ranges that minimally affected cell viability (Fig. 2b), the DR5 agonistic antibody AMG655 significantly reduce the invasion of cancer cells (Fig. 2a). In contrast to DR5 knockdown, AMG655 substantially suppressed TRAF2 polyubiquitination including K63- and K48-specific ubiquitinations, in a concentrationdependent manner (Fig. 2c). Moreover we found that AMG655 decreased TRAF2 levels and enhanced TRAF2 degradation rate (Figs. $2 \mathrm{~d}$ and e), suggesting that
AMG655 destabilizes TRAF2 protein. We also observed that AMG655 caused a delayed reduction of p-JNK and p-c-Jun levels after a transient elevation (Fig. 2f). Collectively, we suggest that AMG655-induced DR5 activation promotes TRAF2 degradation accompanied with a suppression of polyubiquitinaiton and JNK signaling, resulting in eventual suppression of cancer cell invasion.

\section{Sphingosine-1-phosphate (S1P) participates in DR5} knockdown-induced promotion of cell invasion It has been suggested that S1P specifically binds to TRAF2 and regulates its biological functions (e.g., E3 ligase activity) [20, 21]. Thus, we determined whether S1P contributes to TRAF2-mediated promotion of cell

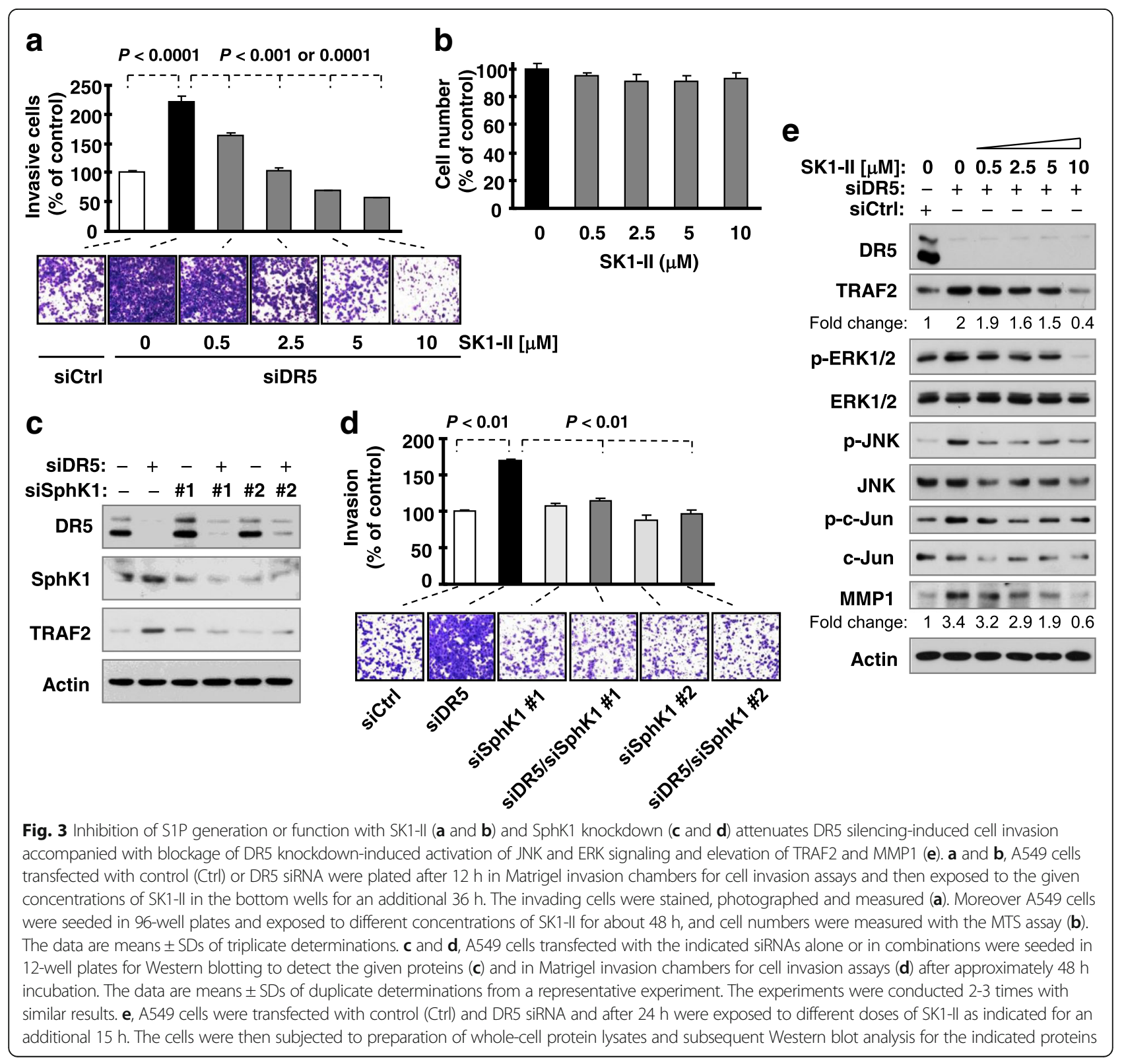


invasion induced by DR5 knockdown. SphK1 is one of the enzymes responsible for the phosphorylation of sphingosine to generate S1P inside cells [22]. Accordingly, SphK1 inhibitors such as SK1-II will decrease intracellular S1P levels. The presence of SK1-II, at concentration ranges that minimally affected cell growth (0.5-10 $\mu \mathrm{M})$, dose-dependently suppressed cell invasion induced by DR5 knockdown (Figs. 3a and b). Similar results were also generated with direct silencing of SphK1 (Figs. 3c and d).

S1P contributes to DR5 suppression-induced elevation of TRAF2 and MMP1 and activation of ERK and JNK signaling

We then determined the involvement of S1P in mediating DR5 knockdown-induced activation of ERK and JNK signaling and activation of TRAF2 and MMP1, required events for DR5 suppression-induced enhancement of invasion [11]. We found that inhibition of S1P with SK1-II blocked the increase in the levels of TRAF2, p-ERK1/2, p-JNK, p-c-Jun and MMP1 induced by DR5 knockdown (Fig. 3e). We also noted that silencing of SphK1 prevented TRAF2 elevation induced by DR5 knockdown (Fig. 3c). These results indicate that inhibition of S1P blocks elevation of TRAF2 and MMP1 and activation of JNK and ERK1/2 signaling. These data again support the involvement of S1P in the promotion of cell invasion induced by DR5 knockdown.

\section{SphK1/S1P signaling is involved in the regulation of TRAF2 polyubiquitination induced by DR5 knockdown}

Given that S1P binds to TRAF2 and regulates E3 ubiquitin ligase activity, particularly for $\mathrm{K} 63$ polyubiquitination [20], we further determined the role of SphK1/S1P signaling in the regulation of TRAF2 polyubiquitination induced by DR5 knockdown. The presence of SKI-II reduced the levels of polyubiquitinated TRAF2 induced by DR5 knockdown (Fig. 4a). Consistently, we detected large amounts of polyubiquitinated TRAF2 in cells transfected with DR5 siRNA, but minimal levels in cells co-transfected with DR5 and SphK1 siRNAs (Fig. 4b). These results clearly show that SphK1/S1P signaling is required for DR5 knockdown-induced enhancement of TRAF2 polyubiquitination.

\section{Caspase-8 regulates TRAF2 polyubiquitination}

In our previous study, we showed that caspase- 8 is important for TRAF2 accumulation, activation of ERK1/2 and JNK/AP-1 signaling and promotion of invasion induced by DR5 knockdown [11]. Here, we asked whether caspase- 8 is also involved in the regulation of TRAF2 polyubiquitination. Hence, we compared TRAF2 polyubiquitination in the absence and presence of caspase- 8 . We detected ubiquitinated TRAF2 in cells cotransfected with TRAF2 and WT, particularly K63 or

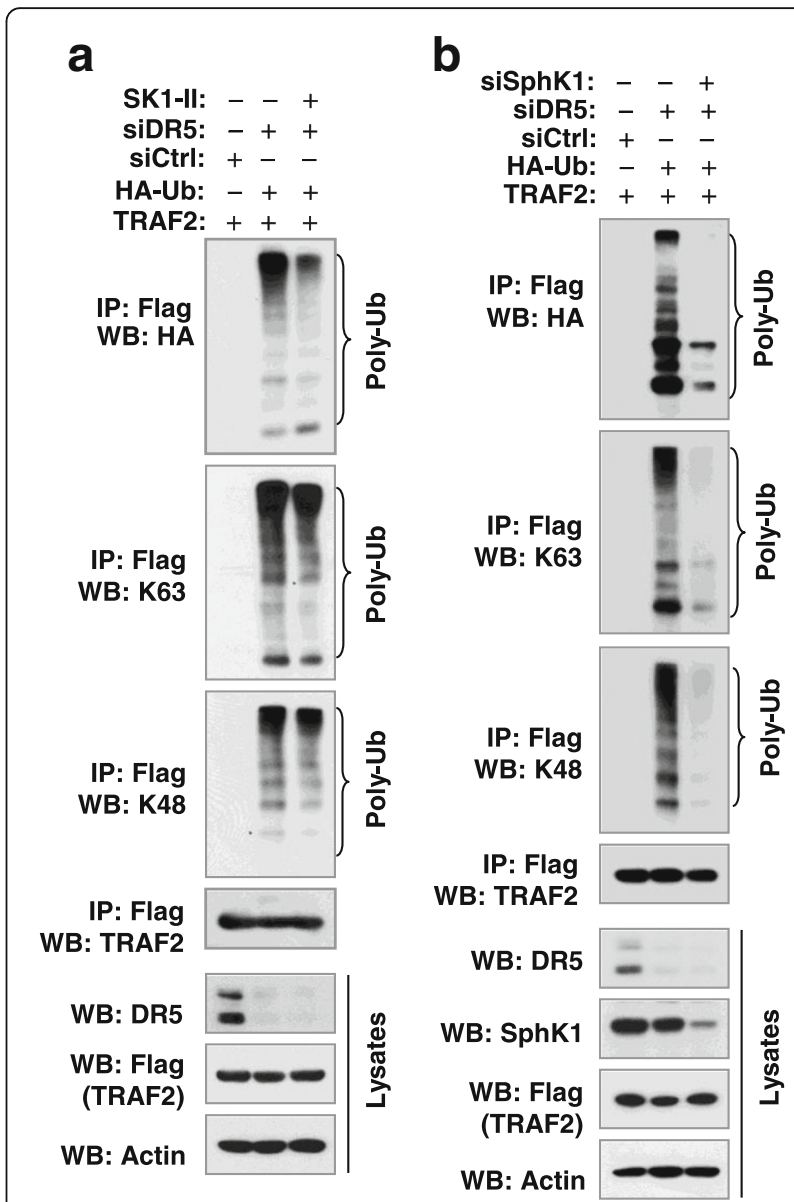

Fig. 4 S1P signaling impacts DR5 knockdown-induced TRAF2 polyubiquitination. $\boldsymbol{a}$ HEK293T cells were co-transfected with the indicated genes or siRNA and after $24 \mathrm{~h}$, were treated with $5 \mu \mathrm{M}$ SK1-II for an additional $10 \mathrm{~h}$. b HEK293T cells were co-transfected with the indicated genes or siRNA and then incubated for $48 \mathrm{~h}$. After the above treatments, whole-cell protein lysates were then prepared from these cells and subjected to IP and subsequent Western blotting (WB) for the indicated proteins

K48 ubiquitin expression plasmids. The levels of these ubiquitinated proteins were substantially enhanced when caspase- 8 was co-expressed (Fig. 5a). In contrast, when endogenous caspase- 8 was depleted by transfection with caspase- 8 siRNA, the levels of these ubiquitinated proteins were diminished (Fig. 5b). These results indicate that caspase-8 regulates TRAF2 polyubiquitination.

We also determined whether caspase- 8 activity is required for regulation of DR5 knockdown-induced TRAF2 polyubiquitination. While knockdown of caspase-8 reduced TRAF2 polyubiquitination induced by DR5 knockdown, enforced expression of both WT and mutant (C360A) caspase-8 elevated TRAF polyubiquitination induced by DR5 siRNA with comparable potencies (Fig. 5c). Since C360A mutation in the caspase domain of caspase- 8 abolishes caspase activity [15], we suggest that caspase activity is not required 

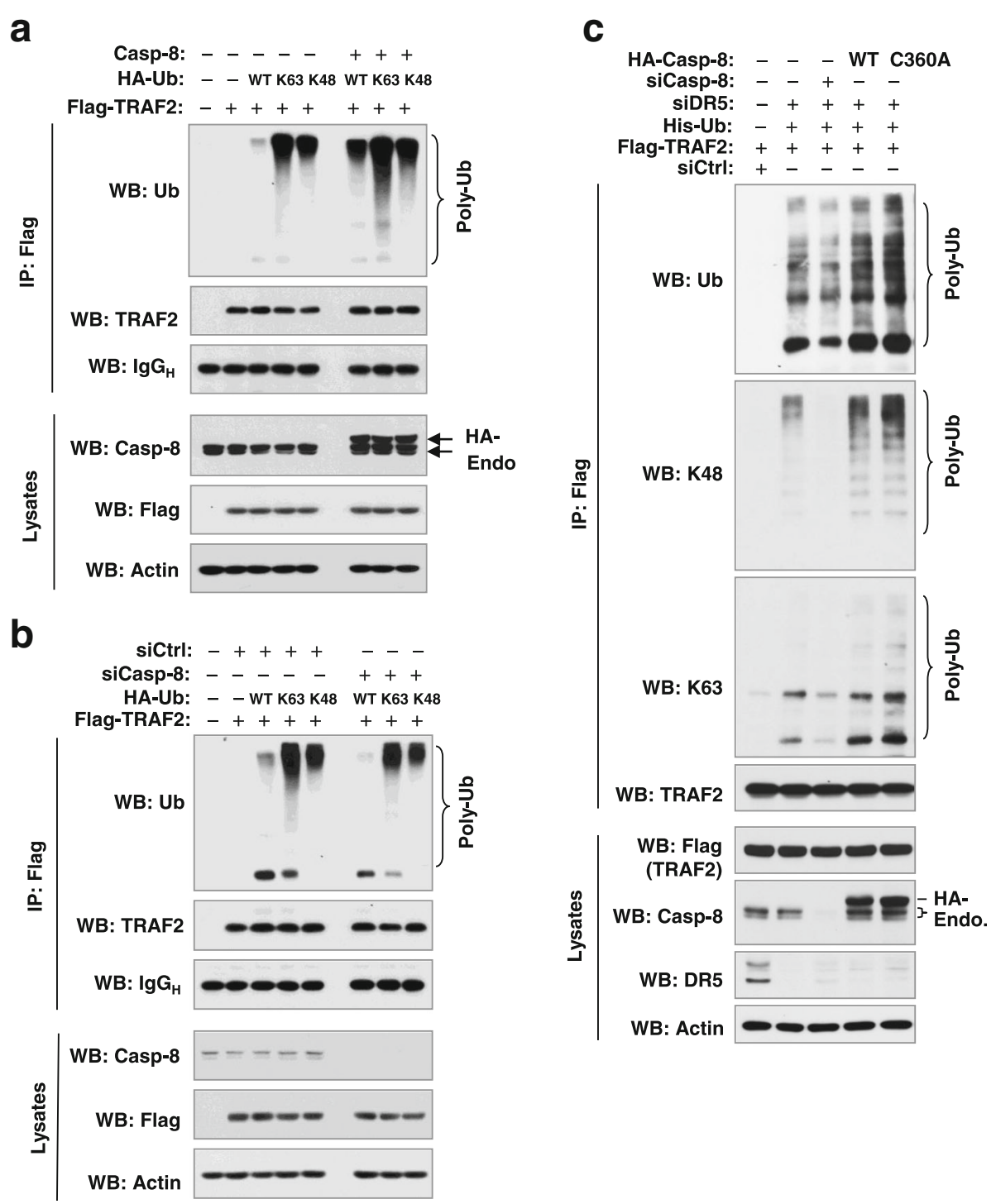

Fig. 5 Caspase-8 modulates TRAF2 polyubiquitination ( $\mathbf{a}$ and $\mathbf{b}$ ) independent of its caspase activity (c). $\mathbf{a}$, HEK293T cells were co-transfected with TRAF2, caspase-8 and WT or mutant Ub plasmid as indicated. b, HEK293T cells were co-transfected with caspase-8 siRNA and plasmids carrying the indicated genes. c, HEK293T cells were co-transfected with Flag-TRAF2 and other indicated genes or siRNAs. After $34 \mathrm{~h}$ of the above transfections, whole-cell protein lysates were prepared from these cells and subjected to IP and subsequent Western blotting (WB) for the indicated proteins

for caspase-8 to modulate DR5 knockdown-induced TRAF2 polyubiquitination.

\section{Discussion}

Involvement of TRAF2 in the positive regulation of cancer cell invasion has been suggested in some previous studies [11, 23, 24]. TRAF2 overexpression has been documented in tumor samples from certain types of cancer such as human pancreatic cancer, breast cancer and gastric cancer, and is associated with cancer progression, metastasis, and shorter patient survival $[23,25,26]$. The current study continues our previous exploration of the critical role of TRAF2-dependent enhancement of cancer cell invasion and metastasis induced by DR5 suppression [11] to further understand the mechanism by which TRAF2 is activated during DR5 suppression-induced promotion of cancer cell invasion.

TRAF2 is known to mediate the activation of both JNK/AP-1 and NF-KB albeit through distinct mechanisms $[27,28]$. However, TRAF2 polyubiquitination, including both $\mathrm{K} 63$ and $\mathrm{K} 48$ ubiquitination, is required for TRAF2 to activate JNK, but not NF-KB [19]. Knockdown of DR5 primarily activates JNK/AP-1 signaling, but not $\mathrm{NF}-\mathrm{kB}$ as demonstrated in our previous report [11]. In this study, we clearly showed that DR5 knockdown increases 
the polyubiquitination of TRAF2 including both $\mathrm{K} 63$ and K48 polyubiquitination (Fig. 1a and b). Moreover, ubiquitination of TRAF2 enhanced AP-1 and MMP1 transcriptional activity including DR5 knockdown-induced transactivation of AP-1 and MMP1 (Fig. 1c). Complementarily, the DR5 agonistic antibody, AMG655, at low concentration ranges that minimally affect cell viability, suppressed TRAF2 polyubiquitination accompanied with enhanced TRAF2 protein degradation and delayed suppression of JNK signaling (Fig. 2). Taken together, these data suggest that TRAF2 polyubiquitination plays an important role in mediating DR5-dependent modulation of cancer cell invasion. Moreover, we have shown that caspase- 8 positively regulates TRAF2 polyubiquitination, since enforced expression of ectopic caspase-8 enhanced TRAF2 polyubiquitination, whereas knockdown of endogenous caspase- 8 expression diminished TRAF2 polyubiquitination (Figs. 5a and b). In agreement with our previous finding that caspase8 enzymatic activity is not required for mediating DR5 suppression-induced cancer cell invasion [11], the current study further demonstrates that caspase- 8 mediates TRAF2 polyubiquitination induced by DR5 suppression independent of its caspase activity (Fig. 5d).

S1P is a pleiotropic lipid mediator that regulates cell growth, cell survival, cell invasion, vascular maturation, and angiogenesis, processes that are important for cancer progression [22]. Although the involvement of S1P in promoting cancer cell invasion and metastasis has been documented in various types of cancers including ovarian, esophageal, prostate, hepatocellular, head and neck, renal, colorectal, breast and pancreatic cancers, glioblastoma and Wilms tumor [29-40], the underlying mechanisms are largely unclear. It is known that S1P exerts most of its biological actions as a specific ligand for a family of five cognate $\mathrm{G}$ protein-coupled receptors in addition to its intracellular functions [22]. S1P has also been suggested to specifically bind to TRAF2 and regulates its biological functions (e.g., E3 ligase activity) [20]. Hence S1P is an essential cofactor for TRAF2 biological activity [21]. We found that inhibition of S1P generation or function with SK1-II or SphK1 knockdown suppressed the enhanced invasion of cancer cells induced by DR5 knockdown, blocked DR5 knockdown-induced activation of ERK1/2 and JNK/AP-1 signaling including elevation of TRAF2 and MMP1, and attenuated DR5 knockdown-induced TRAF2 polyubiquitination (Figs. 3 and 4). Therefore, it appears that S1P is involved in TRAF2-dependent activation of ERK1/2 and JNK/AP-1 signaling and promotion of cell invasion induced by DR5 inhibition. We assume that S1P mediates these processes through binding to TRAF2 independent of S1P receptors although this assumption needs further experimental validation. Our current findings in this regard not only support the role of S1P in the positive regulation of cancer cell invasion and metastasis, but also provide insights into the biology accounting for S1P-dependent promotion of cancer cell invasion and metastasis.

Considering our previous [11] and current findings, we propose a working model as follows: the activation of DR5 favors formation of the death-inducing signaling complex (DISC), resulting in induction of apoptosis or anoikis as well as other potential biological consequences; this not only leads to the direct killing of detached cancer cells (e.g., via anoikis or TRAIL/DR5-mediated immunosurveillance), but also restricts the formation of the metastasis and invasion signaling complex (MISC), eventually resulting in suppression of cancer cell invasion and metastasis. When DR5 is inhibited, cancer cells will be resistant to anoikis or immunosurveillance. Available FADD and caspase- 8 may recruit and stabilize TRAF2; this process will be enhanced by intracellular S1P (e.g., generated by SphK1). Consequently, TRAF2 will be polyubiquitinated and activated, likely through a self-ubiquitination mechanism, resulting in the activation of ERK $1 / 2$ and particularly JNK signaling and subsequent AP-1-dependent expression and activation of MMPs (e.g., MMP1) and finally, promotion of invasion and metastasis of cancer cells (Fig. 6).

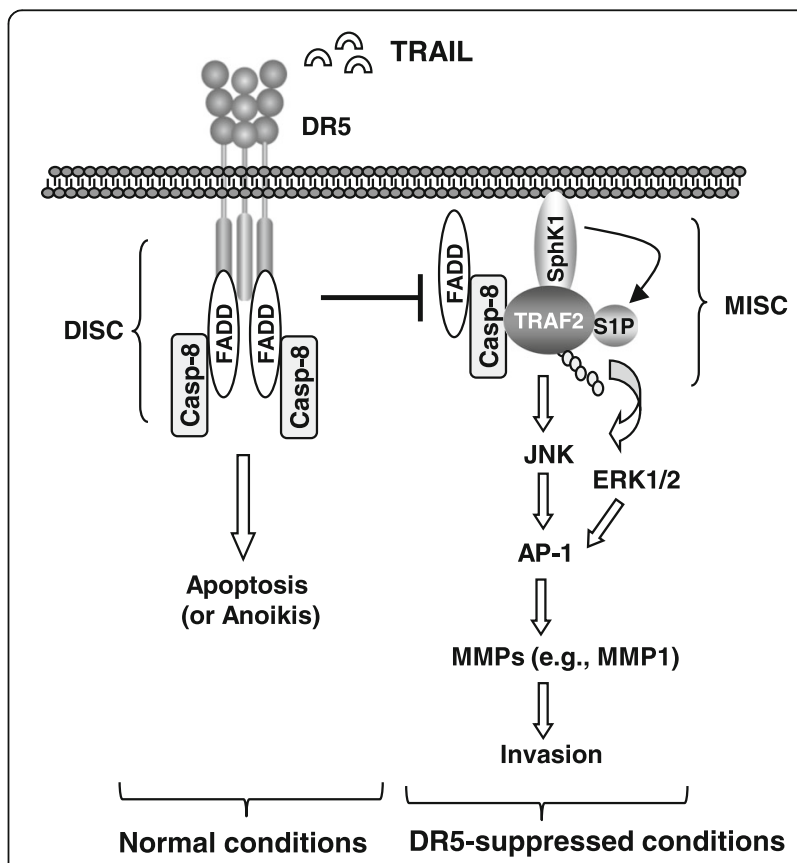

Fig. 6 A working model for DR5-mediated suppression of cancer cell invasion. The primary function of DR5 is to mediate apoptosis upon activation through formation of the DISC; this will restrict the formation of another complex, the metastasis and invasion signaling complex (MISC), and subsequently suppress cell invasion. When DR5 is inhibited, available FADD and caspase-8 may recruit and stabilize TRAF2 with the help of S1P, resulting in enhanced TRAF2 polyubiquitination and activation, likely through a self-ubiquitination mechanism. This will further lead to the activation of ERK and JNK signaling and subsequent AP-1dependent expression and activation of MMPs (e.g., MMP1) and finally, promotion of invasion and metastasis of cancer cells 


\section{Conclusions}

The current study has demonstrated that S1P-dependent TRAF2 polyubiquitination, downstream of caspase-8, is important for mediating DR5 suppression-induced promotion of cancer cell invasion. Together with our previous findings [11], we have highlighted a novel mechanism accounting for enhancement of cancer cell invasion and metastasis caused by DR5 suppression.

\section{Abbreviations}

DR5: Death receptor 5; FADD: Fas-associated death domain; IP: Immunoprecipitation; mDR: Murine death receptor; S1P: Sphingosine-1phosphate; shRNA: Small hairpin RNA; siRNA: Small interfering RNA; SphK2: Sphinogosine kinase 1; TRAF2: Tumor necrosis factor receptorassociated factor 2; WT: Wild-type

\section{Acknowledgements}

We are grateful to Drs. Y.-S. Kim, K. Vuori, H. Habeldah and A.D. Sharrocks for providing plasmids or cell lines. We are also thankful to Dr. A. Hammond in our department for editing the manuscript. Y-T Oh is a recipient of the Emory Winship Cancer Institute Robbins Scholar award. S-Y Sun is an Emory Winship Cancer Institute Halpern Research Scholar and a Georgia Research Alliance Distinguished Cancer Scientist.

\section{Funding}

This study was supported by NIH/NCI SPORE P50 grant CA128613 (to S-Y Sun for Project 2), NIH/NCl cancer center P30 grant CA138292, Emory Winship Cancer Institute Robbins Scholar award (to Y-T Oh) and Emory Winship Cancer Institute Halpern Research Scholar award (to S-Y Sun).

\section{Availability of data and materials}

All data generated or analyzed during this study are included in this article.

\section{Competing interests}

All authors declare no competing interests.

\section{Authors' contributions}

YTO designed and conducted all the experiments. PY prepared reagents and provide technical assistance. SYS conceived and coordinated the study and wrote the paper. All authors reviewed the results and approved the final version of the manuscript.

\section{Consent for publication}

Not applicable.

Ethical approval and consent to participate

Not applicable.

\section{Publisher's Note}

Springer Nature remains neutral with regard to jurisdictional claims in published maps and institutional affiliations.

Received: 23 February 2017 Accepted: 2 May 2017

Published online: 08 May 2017

References

1. Elrod HA, Sun SY. Modulation of death receptors by cancer therapeutic agents. Cancer Biol Ther. 2007;7(2):163-73.

2. Ashkenazi A. Targeting the extrinsic apoptosis pathway in cancer. Cytokine Growth Factor Rev. 2008:19(3-4):325-31.

3. Yang A, Wilson NS, Ashkenazi A. Proapoptotic DR4 and DR5 signaling in cancer cells: toward clinical translation. Curr Opin Cell Biol. 2010;22(6):837-44.

4. Johnstone RW, Frew AJ, Smyth MJ. The TRAIL apoptotic pathway in cance onset, progression and therapy. Nat Rev Cancer. 2008:8(10):782-98.

5. Finnberg N, Klein-Szanto AJ, El-Deiry WS. TRAIL-R deficiency in mice promotes susceptibility to chronic inflammation and tumorigenesis. J Clin Invest. 2008;118(1):111-23.
6. Grosse-Wilde A, Voloshanenko O, Bailey SL, Longton GM, Schaefer U, Csernok Al, Schutz G, Greiner EF, Kemp CJ, Walczak H. TRAIL-R deficiency in mice enhances lymph node metastasis without affecting primary tumor development. J Clin Invest. 2008;118(1):100-10.

7. Zhuang L, Lee CS, Scolyer RA, McCarthy SW, Zhang XD, Thompson JF, Screaton G, Hersey P. Progression in melanoma is associated with decreased expression of death receptors for tumor necrosis factor-related apoptosis-inducing ligand. Hum Pathol. 2006;37(10):1286-94.

8. Elrod HA, Fan S, Muller S, Chen GZ, Pan L, Tighiouart M, Shin DM, Khuri FR, Sun SY. Analysis of death receptor 5 and caspase-8 expression in primary and metastatic head and neck squamous cell carcinoma and their prognostic impact. PLoS One. 2010;5(8):e12178.

9. Shin MS, Kim HS, Lee SH, Park WS, Kim SY, Park JY, Lee JH, Lee SK, Lee SN, Jung SS, et al. Mutations of tumor necrosis factor-related apoptosis-inducing ligand receptor 1 (TRAIL-R1) and receptor 2 (TRAIL-R2) genes in metastatic breast cancers. Cancer Res. 2001;61(13):4942-6.

10. Malin D, Chen F, Schiller C, Koblinski J, Cryns VL. Enhanced metastasis suppression by targeting TRAIL receptor 2 in a murine model of triplenegative breast cancer. Clin Cancer Res. 2011:17(15):5005-15.

11. Oh YT, Yue P, Wang D, Tong JS, Chen ZG, Khuri FR, Sun SY. Suppression of death receptor 5 enhances cancer cell invasion and metastasis through activation of caspase-8/TRAF2-mediated signaling. Oncotarget. 2015;6(38):41324-38.

12. Hoogwater FJ, Nijkamp MW, Smakman N, Steller EJ, Emmink BL, Westendorp BF, Raats DA, Sprick MR, Schaefer U, Van Houdt WJ, et al. Oncogenic K-Ras turns death receptors into metastasis-promoting receptors in human and mouse colorectal cancer cells. Gastroenterology. 2010;138(7):2357-67.

13. von Karstedt S, Conti A, Nobis M, Montinaro A, Hartwig T, Lemke J, Legler K, Annewanter F, Campbell AD, Taraborrelli $L$, et al. Cancer cell-autonomous TRAIL-R signaling promotes KRAS-driven cancer progression, invasion, and metastasis. Cancer Cell. 2015;27(4):561-73.

14. Liu X, Yue P, Zhou Z, Khuri FR, Sun SY. Death receptor regulation and celecoxib-induced apoptosis in human lung cancer cells. J Natl Cancer Inst. 2004;96(23):1769-80

15. Finlay D, Vuori K. Novel noncatalytic role for caspase-8 in promoting SRCmediated adhesion and Erk signaling in neuroblastoma cells. Cancer Res. 2007:67(24):11704-11.

16. Thomas GS, Zhang L, Blackwell K, Habelhah H. Phosphorylation of TRAF2 within its RING domain inhibits stress-induced cell death by promoting IKK and suppressing JNK activation. Cancer Res. 2009;69(8):3665-72.

17. Guan B, Yue P, Lotan R, Sun SY. Evidence that the human death receptor 4 is regulated by activator protein 1. Oncogene. 2002;21(20):3121-9.

18. Pfahl M, Tzukerman M, Zhang XK, Lehmann JM, Hermann T, Wills KN, Graupner G. Nuclear retinoic acid receptors: cloning, analysis, and function. Methods Enzymol. 1990;189:256-70.

19. Habelhah H, Takahashi S, Cho SG, Kadoya T, Watanabe T, Ronai Z Ubiquitination and translocation of TRAF2 is required for activation of JNK but not of p38 or NF-kappaB. EMBO J. 2004;23(2):322-32.

20. Alvarez SE, Harikumar KB, Hait NC, Allegood J, Strub GM, Kim EY, Maceyka $M$, Jiang $H$, Luo $C$, Kordula $T$, et al. Sphingosine-1-phosphate is a missing cofactor for the E3 ubiquitin ligase TRAF2. Nature. 2010;465(7301):1084-8.

21. Napolitano G, Karin M. Sphingolipids: the oil on the TRAFire that promotes inflammation. Sci Signal. 2010;3(141):e34.

22. Milstien S, Spiegel S. Targeting sphingosine-1-phosphate: a novel avenue for cancer therapeutics. Cancer Cell. 2006;9(3):148-50.

23. Trauzold A, Roder C, Sipos B, Karsten K, Arlt A, Jiang P, Martin-Subero الر, Siegmund D, Muerkoster S, Pagerols-Raluy L, et al. CD95 and TRAF2 promote invasiveness of pancreatic cancer cells. FASEB J. 2005;19(6):620-2.

24. Jang KW, Lee KH, Kim SH, Jin T, Choi EY, Jeon HJ, Kim E, Han YS, Chung JH. Ubiquitin ligase CHIP induces TRAF2 proteasomal degradation and NFkappaB inactivation to regulate breast cancer cell invasion. J Cell Biochem. 2011;112(12):3612-20.

25. Zhao ZJ, Ren HY, Yang F, Wang J, Wu GP, Mi XY. Expression, correlation, and prognostic value of TRAF2 and TRAF4 expression in malignant plural effusion cells in human breast cancer. Diagn Cytopathol. 2015:43(11):897-903.

26. Zhang J, Dai WJ, Yang XZ. Methylation status of TRAF2 is associated with the diagnosis and prognosis of gastric cancer. Int J Clin Exp Pathol. 2015;8(11):14228-34

27. Reinhard C, Shamoon B, Shyamala V, Williams LT. Tumor necrosis factor alpha-induced activation of c-jun N-terminal kinase is mediated by TRAF2. EMBO J. 1997;16(5):1080-92.

28. Bradley JR, Pober JS. Tumor necrosis factor receptor-associated factors (TRAFs). Oncogene. 2001;20(44):6482-91. 
29. Van Brocklyn JR, Young N, Roof R. Sphingosine-1-phosphate stimulates motility and invasiveness of human glioblastoma multiforme cells. Cancer Lett. 2003;199(1):53-60.

30. Smicun $Y$, Reierstad S, Wang FQ, Lee C, Fishman DA. S1P regulation of ovarian carcinoma invasiveness. Gynecol Oncol. 2006;103(3):952-9.

31. Park KS, Kim MK, Lee HY, Kim SD, Lee SY, Kim JM, Ryu SH, Bae YS. S1P stimulates chemotactic migration and invasion in OVCAR3 ovarian cancer cells. Biochem Biophys Res Commun. 2007;356(1):239-44.

32. Miller AV, Alvarez SE, Spiegel S, Lebman DA. Sphingosine kinases and sphingosine-1-phosphate are critical for transforming growth factor betainduced extracellular signal-regulated kinase 1 and 2 activation and promotion of migration and invasion of esophageal cancer cells. Mol Cell Biol. 2008;28(12):4142-51.

33. Li MH, Sanchez T, Yamase H, Hla T, Oo ML, Pappalardo A, Lynch KR, Lin CY, Ferrer F. S1P/S1P1 signaling stimulates cell migration and invasion in Wilms tumor. Cancer Lett. 2009;276(2):171-9.

34. Sekine Y, Suzuki K, Remaley AT. HDL and sphingosine-1-phosphate activate stat3 in prostate cancer DU145 cells via ERK1/2 and S1P receptors, and promote cell migration and invasion. Prostate. 2011;71(7):690-9.

35. Tamashiro PM, Furuya H, Shimizu Y, Kawamori T. Sphingosine kinase 1 mediates head \& neck squamous cell carcinoma invasion through sphingosine 1-phosphate receptor 1. Cancer Cell Int. 2014;14(1):76

36. Salama MF, Carroll B, Adada M, Pulkoski-Gross M, Hannun YA, Obeid LM. A novel role of sphingosine kinase-1 in the invasion and angiogenesis of $\mathrm{VHL}$ mutant clear cell renal cell carcinoma. FASEB J. 2015;29(7):2803-13.

37. Long J, Xie Y, Yin J, Lu W, Fang S. SphK1 promotes tumor cell migration and invasion in colorectal cancer. Tumour Biol. 2016;37(5):6831-6.

38. Li J, Song Z, Wang Y, Yin Y, Liu Y, Yuan R, Nan X. Overexpression of SphK1 enhances cell proliferation and invasion in triple-negative breast cancer via the PI3K/AKT signaling pathway. Tumour Biol. 2016;37(8):10587-93.

39. Ko P, Kim D, You E, Jung J, Oh S, Kim J, Lee KH, Rhee S. Extracellular matrix rigidity-dependent sphingosine-1-phosphate secretion regulates metastatic cancer cell invasion and adhesion. Sci Rep. 2016;6:21564.

40. Guo YX, Ma YJ, Han L, Wang YJ, Han JA, Zhu Y. Role of sphingosine 1phosphate in human pancreatic cancer cells proliferation and migration. Int J Clin Exp Med. 2015;8(11):20349-54.

\section{Submit your next manuscript to BioMed Central and we will help you at every step:}

- We accept pre-submission inquiries

- Our selector tool helps you to find the most relevant journal

- We provide round the clock customer support

- Convenient online submission

- Thorough peer review

- Inclusion in PubMed and all major indexing services

- Maximum visibility for your research

Submit your manuscript at www.biomedcentral.com/submit

) Biomed Central 